\title{
El testimonio de Joaquina Grefa, una cautiva quichua entre los huaorani (Ecuador, 1945)
}

\section{María Susana Cipolletti}

\section{(2) OpenEdition \\ 12 Journals}

Edición electrónica

URL: http://journals.openedition.org/jsa/2759

DOI: $10.4000 /$ jsa.2759

ISSN: 1957-7842

\section{Editor}

Société des américanistes

\section{Edición impresa}

Fecha de publicación: 1 enero 2002

Paginación: 111-135

ISSN: 0037-9174

\section{Referencia electrónica}

María Susana Cipolletti, «El testimonio de Joaquina Grefa, una cautiva quichua entre los huaorani (Ecuador, 1945) », Journal de la société des américanistes [En línea], 88 | 2002, Publicado el 05 enero 2007, consultado el 19 abril 2019. URL : http://journals.openedition.org/jsa/2759; DOI : 10.4000/ jsa. 2759 


\title{
EL TESTIMONIO DE JOAQUINA GREFA, UNA CAUTIVA QUICHUA ENTRE LOS HUAORANI (ECUADOR, 1945) ${ }^{1}$
}

\author{
María Susana CIPOLLETTI *
}

En 1944 los huaorani raptaron a una niña quichua, que escapó un año más tarde y relató lo vivido alli a un misionero inglés. En esa época, los huaorani mantenían relaciones exclusivamente agresivas con la sociedad circundante, por lo cual no se conocen hasta ahora informes de testigos, lo que hace del testimonio de Joaquina un documento excepcional. Este refleja distintos aspectos de la vida huao, incluyendo las formas de la guerra.

Palabras Clave: historia de vida, relaciones interétnicas, guerra, etnohistoria, huaorani, Ecuador.

The Joaquina Grefa's testimony, a Quichua girl kidnapped by Huaorani (Ecuador, 1945)

In 1944, a group of Huaorani kidnapped a Quichua girl, who escaped a year later and narrated the story of her life among the Huaorani to an English missionary. Back then, relations between the Huaorani and the surrounding society were exclusively aggressive. For that reason, there were no eyewitness accounts. This converts Joaquina's testimony in an extraordinary document, reflecting different aspects of Huaorani life, including forms of war.

KEY WORDS : life-history, interethnic relations, war, ethnohistory, Huaorani, Ecuador.

\section{Le témoignage de Joaquina Grefa, captive quechua chez les Waoranis (Équateur, 1945)}

En 1944, un groupe de Waoranis enleva une jeune fille quechua qui réussit à leur échapper un an plus tard. Elle raconta à un missionnaire anglais sa vie parmi ces Indiens. À cette époque, les Waoranis entretenaient des relations exclusivement agressives avec la société environnante, ce qui explique l'absence d'informations extérieures sur ce groupe, faute de témoignages directs. Le récit de Joaquina revêt dès lors un caractère exceptionnel, car il décrit différents aspects de la vie quotidienne des Waoranis, y compris les actes de guerre.

MотS Clés : récit de vie, relations interethniques, guerre, ethnohistoire, Waorani, Équateur.

* Institut für Altamerikanistik und Ethnologie, Universität Bonn, Römerstrasse 164, D-53117 Bonn, E-mail : m.cipolletti@uni-bonn.de

Journal de la Société des Américanistes, 2002, 88 : p. 111 à 135. Copyright (C) Société des Américanistes. 


\section{INTRODUCCIÓN}

Numerosas sociedades indígenas sudamericanas practican el rapto de mujeres o la toma de cautivas a nivel intraétnico, pero pocas sociedades lo hicieron a nivel interétnico. El más famoso de los relatos en los que la sociedad indígena aparece desde el punto de vista de una cautiva es el de Helena Valero (1984), que fue raptada por los yanomami en Venezuela a fines de 1940. Mientras que en este sector los casos fueron muy esporádicos, el número de cautivas quichua entre los huaorani entre 1940 y 1960 y en relación al tamaño de los grupos, fue considerable, aunque no sea posible dar una cifra exacta. El rol que las cautivas tenían entre los huaorani se ve con claridad a través de la comparación con el rol de las cautivas en otras sociedades, por lo cual aludiremos también más adelante a las modalidades del cautiverio en uno de los casos más notables y mejor documentados de América del Sur : el de las sociedades de Pampa y Patagonia.

El informe al que me referiré a continuación revela los entresijos de un tema central en esta sociedad, cuya belicosidad fue la más notoria de América del Sur (Robarchek \& Robarchek 1998, Yost 1981). En las últimas décadas se han multiplicado las teorías y perspectivas acerca de los motivos de la guerra a escala mundial (véase Orywal 1996). Si bien no trato aquí en detalle la guerra huao, la influencia del frente de colonización, que provocó una intensificación y transformación de la misma, fue decisiva. Lo observado por Joaquina durante su estadía entre los huaorani, corrobora ampliamente la perspectiva de Ferguson y Whitehead (1999) acerca de la influencia ejercida por la presencia occidental sobre la guerra indígena. La existencia de un frente de colonización o, más bien, la comprensión de la magnitud numérica y tecnológica del mismo fue, en mi opinión, un motivo decisivo para que los huaorani aceptaran la pacificación a partir de 1960-1970.

De las primeras décadas del siglo $\mathrm{xx}$ datan informaciones sobre los conflictos de los huaorani con el frente de penetración en la región oriental del Ecuador - trabajadores de las prospecciones geológicas y petroleras, colonos, campesinos. Relatos de pillajes por parte de los huaorani, venganzas por muertes acaecidas en uno y otro bando y los raptos mutuos de individuos son parte de la historia y las leyendas delárea.

En la región bajo consideración, los quichua eran en su mayoría trabajadores de haciendas, que, o bien en incursiones al territorio huao, o bien en represalia de un pillaje, capturaban a huaorani. Se apoderaban preferentemente de niños de uno u otro sexo o de muchachas las cuales, en la mayoría de los casos, terminaban como "trabajadores » en haciendas de la región o, en algún caso, como empleadas de servicio doméstico en Quito. Estos casos se hallan documentados aproximadamente a partir de 1900 (Cabodevilla 1994, pp. 192, 214, 245 ; Stoll 1982, p. 281). La condición de los huaorani como trabajadores en las haciendas se asemejaba más a la de siervos y esclavos que lo que solemos entender con esa denominación. Con la entrega de varias muchachas huaorani a un patrón de conspicua aparición en las fuentes (Carlos Sevilla), su captor saldó con él una deuda monetaria (Dall'Alba 1992, p. 106), lo que muestra que los huaorani tenían un valor definido. Mientras que a los patrones les interesaba la fuerza de trabajo gratis que aquellos representaban, la orden católica de los josefinos, activa en el área, también mostraba interés - aunque por motivos muy 
diferentes - , pues esperaban educar a algunos niños para que actuaran de mediadores en contactos futuros (Cabodevilla 1994, p. 243). Los huaorani apresados no sobrevivieron por lo general a las condiciones de vida lejos de su grupo, enfermaron gravemente o cayeron en un estado de apatía y depresión, como dos mujeres presas en un claustro en Quito o, en un acto de desesperación, se suicidaron ingiriendo barbasco (veneno para peces ; véase Blomberg 1996, pp. 48 ss., 54 ss.).

Con el recrudecimiento de los conflictos internos entre los huaorani alrededor de 1950, varias mujeres huyeron hacia el exterior. La fuga más famosa fue la de Dayuma, pues abrió las puertas a la misión del Instituto Lingüístico de Verano (ILV/SIL), luego que la misionera Rachel Saint la liberara de su destino en la hacienda Illa y regresara con ella al grupo huao. El coraje de las mujeres que huían de la situación desesperada que vivían en el seno de su sociedad, se patentiza aún más al considerar que, en opinión de los huaorani, los quichua, los mestizos y los blancos eran caníbales (Rival 1996a, p. 71 ; Wallis 1996 , p. 49) ${ }^{2}$.

La toma de cautivos y su incorporación violenta era así común a ambas sociedades. Sin embargo, el fenómeno presenta una diferencia esencial : las mujeres huaorani eran incorporadas al estrato más bajo de la sociedad regional y de los trabajadores de la hacienda - previa violación, que era parte de la incorporación a ese nuevo mundo. Por el contrario, la situación de las cautivas quichua, como veremos en el relato de Joaquina, no parece haberse diferenciado en nada de la de otras mujeres del grupo. Eso sí, los huaorani tomaron las medidas necesarias para que se asemejara a ellos : le quitaron la ropa, le agujerearon los lóbulos de las orejas y, el mismo día en que la raptaron, le dieron una hamaca — en realidad, dos, pues la niña, enfurecida, arrojó la hamaca al río... actitud que no logró irritar a sus captores, que esa misma noche le entregaron otra (Tidmarsh \& Grefa 1945, pp. 1, 5). Las informaciones existentes no dan pie a la afirmación de Robarchek (1998, p. 98) de que la expectativa de vida de las cautivas era breve, pues las mataban luego de unos meses : varias cautivas vivieron muchos años con los huaorani, y por lo menos una antigua cautiva sigue viviendo allí en la actualidad, casada con un viejo guerrero (St. Beckerman, comunicación verbal, enero 2001, foto en Cabodevilla 1994, p. 454).

El grupo con el que vivió Joaquina estaba compuesto por 32 personas, de las cuales 18 eran mujeres : además de Joaquina, había otras dos mujeres quichua : Margarita y Api (Victoria), la cual había sido raptada de pequeña y huyó luego con Dayuma en 1947 (Cabodevilla 1994, p. 262 ; Wallis 1996, p. 58) ${ }^{3}$. Sobre Margarita no hemos encontrado más informaciones.

Años más tarde, en 1957, vivió en este mismo grupo una muchacha llamada Maruja Huatatoca, raptada cuando tenía 18 años, luego de que asesinaran a su esposo. Su liberación se debió a la influencia ejercida por el Instituto Lingüístico de Verano, cuando dos mujeres huaorani regresaron a su grupo luego de una larga ausencia y convencieron al esposo huao de Maruja de que la liberara (Elliot 1989, pp. 29, 57 ; véase también Cabodevilla 1994, pp. 315, 335).

El último caso de cautiverio del que tenemos noticia se halla documentado en el relato de un guerrero huao : en 1964 su grupo atacó a una familia quichua y robó a una niña de unos 5 años, que vivió varios años con ellos y con otro niño raptado anteriormente. También en este caso los dos cautivos fueron liberados bajo la presión de Dayuma (Patzelt 1992, p. 64). 
Los casos de varones cautivos son muy escasos. Además del niño que acabamos de mencionar, se cuenta con Sebastián Shihuango, que tenía unos 7 años cuando sus padres fueron asesinados en 1947 en el río Arajuno y vivió diez años con los huaorani antes de regresar con su familia (Baumann \& Patzelt 1982, pp. 21 ss.). La excepcionalidad del rapto de varones se explica probablemente por el imperativo huao, de que, al crecer; los hijos varones deben vengar el asesinato de sus padres. Por este motivo, un niño quichua era potencialmente un enemigo.

La alteridad entre ambas sociedades indígenas era muy marcada : los huaorani concebían a todo extraño al grupo como un eventual caníbal e inspiraban a su vez terror a los quichua con sus silenciosos desplazamientos por la selva, sus ataques sorpresivos y su veloz retirada. Entre los quichua empleados por las compañías petroleras circulaba el rumor que no se los podía matar con armas de fuego y que arrojaban ayaallpa (tierra sacada de antiguos entierros) a los trabajadores, para sumirlos en un profundo sueño y poder atacarlos impunemente (Muratorio 1987, pp. 169 s.). Los quichua negaban a los huaorani el don del lenguaje - o sea, la condición de seres humanos - ya que no reconocían su idioma como habla humana y opinaban que aquellos « sólo producen sonidos » o que « sólo hacían ruidos como animales » (Elliot 1989, p. 33 ; Blomberg 1996, p. 54). En las últimas décadas las relaciones entre individuos de ambas sociedades han cambiado de signo e incluyen transacciones comerciales y sociales, como compadrazgos y casamientos. Yost (1981b, pp. $700 \mathrm{~s}$.) analiza las relaciones en los años 70 , que presentan diferencias también con respecto a las actuales. Si bien los quichua siguen viendo a los huaorani como « salvajes », la opinión de las mujeres quichua es favorable, pues los huaorani son excelentes cazadores, no castigan a las mujeres ni les exigen grandes trabajos. Para suegros potenciales, tener a un yerno o una nuera huao implica el acceso a su territorio y, así, a sus ricos recursos naturales (Rival 1996a, p. 178).

\section{LOS PROTAGONISTAS}

A continuación me referiré a las sociedades protagonistas de esta historia sólo en la medida necesaria para enmarcar el relato de Joaquina. « Auca » es una palabra quichua que significa « salvaje », y se aplicó desde la época colonial hasta la actualidad a distintos grupos étnicos en diferentes regiones. En el Ecuador los huaorani recibieron esta denominación hasta hace unos diez años, cuando, en parte a su instancia, se suplantó por el etnónimo propio : huao, " persona » (singular), huaorani (plural).

La bibliografía sobre esta etnia consiste sobre todo en relatos de viajes de aventura y expediciones, inspiradas por esta sociedad elusiva, guerrera y que en opinión de los extraños vivía aún en "la edad de piedra ». Los trabajos antropológicos son aún escasos y pueden reducirse a tres autores: James Yost (1981a, 1981b, 1994), Laura Rival (1993, 1996a, 1996b, 1998, 1999) y Miguel Angel Cabodevilla (1994). Más que realizar un análisis etnográfico, Cabodevilla ubica la historia huao en un contexto nacional y señala las intrincadas relaciones e influencias entre ambas, utilizando un vasto número de fuentes, que incluyen documentos de archivos regionales y grabaciones magnetofónicas de miembros de la orden capuchina.

La proveniencia geográfica y la filiación cultural de los huaorani son poco claras. Recién a partir de principios del siglo $\mathrm{xx}$, con las incipientes incursiones en su 
territorio, se tomó conocimiento de su existencia. Aunque éste no es el lugar para una discusión extensa de la filiación huao, hay que señalar que diferentes autores los identifican como descendientes de los (hoy desaparecidos) abijira o auishiri, basándose en el hecho de que habitaban el mismo territorio. A partir de esta premisa, atribuyen a los huaorani los rasgos culturales auishiri que apuntó Tessmann (1930, pp. 475-486), sin tener en cuenta que el vocabulario auishiri que trae este autor es claramente no huao. En cambio, sí lo es el vocabulario procedente de un grupo al que denomina « Sabela » (Tessmann 1930, pp. 298-302) ; denominación que, sin embargo, no había logrado aclarar el panorama, ya que no era posible relacionarla con ninguna sociedad de la región. Su origen ha sido explicado por Cabodevilla (1994, p. 258), basándose en la obra del viajero Loch : Sabela proviene de « Isabel », un nombre dado a una mujer huao que alrededor de 1910 tuvo contactos con los empleados de una hacienda de la región, quienes utilizaron este nombre para denominar a todo el grupo.

La desaparición de los auishiri, que en el pasado habitaban parte del territorio actual huao, puede atribuirse a que fueron en parte aniquilados por los huaorani y formaron una de las corrientes que confluyeron en la etnogénesis de la actual etnia quichua de las tierras bajas. Otros hechos apuntalan asimismo la no-identidad auishiri/huaorani ${ }^{4}$.

Es probable que el intento de identificar en la región a una sociedad que sea antecesora directa de la actual sociedad huao esté condenado al fracaso, no sólo por la ausencia de fuentes sino porque probablemente no hubo una sola antecedente directa. Además, hasta ahora no se ha logrado demostrar un parentesco lingüístico con otras lenguas de la región. En unas pocas páginas, aparecidas como colofón a una publicación, Zerries (1982, p. 158) menciona brevemente distintas posibilidades para la filiación huao y sugiere que quizás se trate de un resto de distintos grupos que formaron secundariamente una unidad. Si bien Zerries no fundamenta su opinión, probablemente esté en lo cierto. Mi hipótesis va también en esa dirección y se basa en consideraciones etnohistóricas y etnográficas. Las misiones jesuíticas de Maynas, cuyo centro se hallaba en la selva del Perú actual, no lograron jamás controlar a la totalidad de los grupos. Aún más, las fugas de grupos de indígenas de las misiones eran tan habituales que se montaban frecuentes expediciones para obligarlos a volver por medio de la violencia. Cuando los jesuitas fueron expulsados en 1767, existían numerosas sociedades " cimarronas » que habían obtenido nichos en el hinterland fuera de la esfera de influencia de aquellos. Una zona de refugio fue el río Pastaza, donde a mediados del siglo XVII se habían ocultado algunos grupos ${ }^{5}$. Una hipótesis plausible es que estos grupos poco numerosos fueron remontando los ríos, evitando el contacto con los centros poblados, mezclándose, y dieron origen a los huaorani. Su condición de prófugos, su situación de defensa continua ante otros grupos, podría haber originado en parte su actitud contestataria. Su procedencia de la región de los contrafuertes andinos explicaría también la ausencia de algunos rasgos característicos de las sociedades amazónicas (ver discusión en Cabodevilla 1994, p. 37).

Por su parte, los quichua selváticos, quichua del Napo o naporuna (runa : « persona ») son resultado de una expansión poblacional en la región y de un proceso de etnogénesis, cuya compleja filiación y absorción de otros grupos han sido tratadas por varios autores (Cabodevilla 1994, pp. 109, 145 ss. ; Hudelson 1984, p. 60 ; Muratorio 1987, pp. 45 ss. ; Oberem 1980 ; Whitten 1976, pp. 7 ss.). A menudo se los llamó 


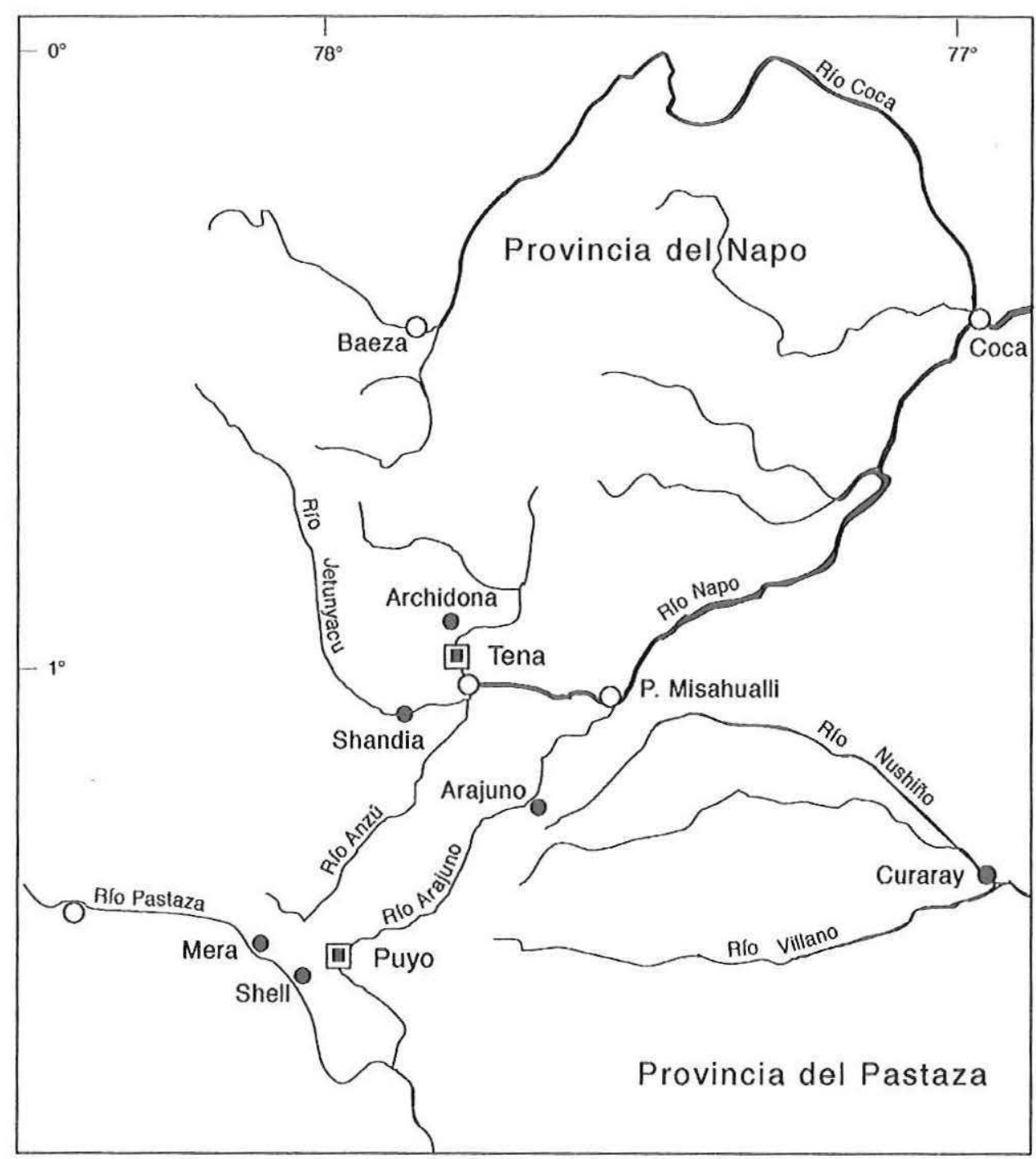

Fig. 1. - Región entre los Rios Pastaza y Napo.

incorrectamente "yumbos ", denominación tomada de un grupo que vivía en las cercanías del volcán Pichincha (Oberem 1980, pp. 31 ss.), y que es la utilizada con respecto a las cautivas quichua en el documento que presentaremos.

Veamos unos pocos datos de la compleja historia del desarrollo de la región del Napo (véase Figura 1), necesarios para ubicar el relato de Joaquina. El inicio de la colonización de la región del río Napo, seguida por la explotación petrolera, data de 1920-1930. En 1938 llegan geólogos europeos y norteamericanos a Mera (en la actualidad Shell-Mera), que hacen construir una pista de aterrizaje y comienzan a 


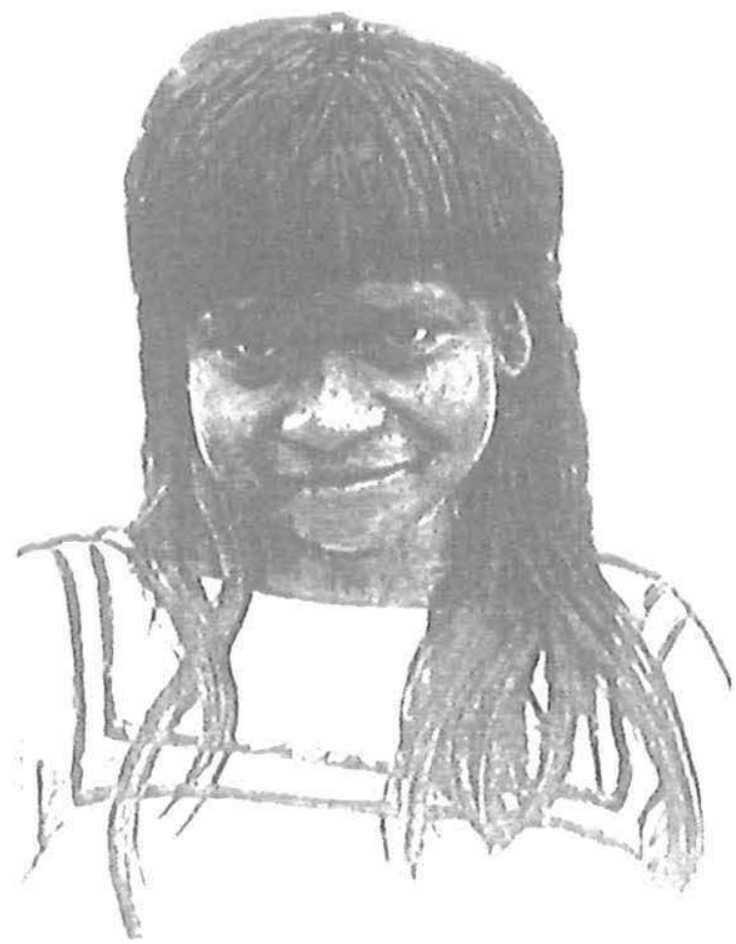

FIG. 2. - Joaquina Grefa después de su cautiverio

cartografiar la enorme región otorgada en concesión. A partir de esta época se suceden los ataques huaorani a los campamentos (Cabodevilla 1994, pp. 218 ss. 284 ss. ; Muratorio 1987).

Dada la ausencia de caminos en la región y la navegabilidad parcial de los ríos, la comunicación se realizaba a través del tráfico aéreo, en parte con aviones de gran tamaño. Estos proveían de lo necesario a los campamentos de Napo y Pastaza, que carecían de pista de aterrizaje : hacían descender desde el aire víveres y hasta jeeps y camiones - e incluso animales, como lo demuestra la curiosa foto de los « cerdos paracaidistas » tomada por Blomberg (1996). Como veremos, el intenso tráfico aéreo impresionó profundamente a los huaorani, pues implicaba el dominio de una esfera que estaba fuera de su control. Esto se exacerbó cuando la misión protestante comenzó a arrojar objetos desde el aire y dio lugar a una peculiar concepción de los aviones y una suerte de cargo-cult.

\section{JOAQUINA GREFA ${ }^{6} \mathrm{Y}$ SU MISTERIOSO INTERROGADOR}

Los archivos son instituciones elusivas y a menudo los documentos se encuentran o por casualidad o por un revés de la suerte, como en este caso. Circunstancias adversas me impidieron realizar la segunda parte de mi investigación de campo, por lo 
cual me dediqué a la investigación en archivos, entre otros, del Archivo Histórico del Banco Central de Quito, donde encontré, entre los manuscritos no catalogados, el documento que publico aquí ${ }^{7}$. En 1945, fecha de su redacción, no existían contactos pacíficos entre los huaorani y el mundo circundante, por lo cual no había ni observadores ni visitantes - excepción hecha de las cautivas. Joaquina vivió en una casa aliada a Moipa, un guerrero al cual se le atribuye la mayor parte de los pillajes y asesinatos de las décadas del 40 y el 50 y que tuvo un papel protagónico en las agresiones internas, por lo cual es una figura clave para entender la guerra.

El documento aquí transcrito lleva por título Unarranged Notes on the Aucas of the Nushino Basin (Tidmarsh \& Grefa 1945) y consta de ocho páginas numeradas, escritas en inglés a máquina. La primera página lleva por título adicional « Miscellaneous Unarranged Notes on the Aucas of Eastern Ecuador ». El segundo documento, (que no transcribo), Vocabulario tentativo del Idioma de Los Aucas con unas Frases, consta asimismo de ocho páginas escritas a máquina, y recoge un vocabulario en castellano, quichua del oriente y « auca », compuesto por 230 voces y unas 40 frases, clasificadas por el autor en : partes del cuerpo, objetos, fenómenos naturales, comida y plantas, vida animal, pájaros, pescados, misceláneas y palabras no clasificadas (Tidmarsh 1945). Acompaña a los documentos una foto en blanco y negro de Joaquina, reproducida aquí. Al reverso se lee, escrito a mano : « Niña yumba que fue capturada por los aucas y que escapó de sus manos después de un año de cautividad, y me suministró los datos aquí escritos. W. J. T. 29.6.45 ». Ambos documentos se hallan en el Archivo Histórico del Banco Central, Quito, Col. 24, con las siglas respectivas 01308 y $01411^{8}$.

Hasta ahora he preferido referirme sin más precisiones al " autor », ya que en los documentos no aparece su nombre completo sino solamente las iniciales W. J. T. La búsqueda en la bibliografía de nombres de viajeros y misioneros que se hallaban en esos años en la región permite identificarlo como el pastor inglés Wilfred Tidmarsh. Elizabeth Elliot, que fue misionera entre los huaorani, a quien planteé esta pregunta, es de la misma opinión (E. Elliot, carta a M.S.C., diciembre 2001). Cabodevilla (1994, p. 318) cita además un artículo en un diario quiteño de la década del 50, que lamenta que Tidmarsh no publique sus escritos?

Tidmarsh era doctor en filosofia, había estudiado homeopatía y vivía desde 1939 en el Ecuador. En 1950 dirigía una misión quichua en Shandia, en la cual se asentaron los misioneros protestantes que en 1956 fueron masacrados por los huaorani (Elliot 1981, pp. 23, 43). Luego de la matanza, Tidmarsh intentó otro tipo de acercamiento, para lo cual construyó una choza en las orillas del Curaray, donde dejó distintos objetos y comenzó a construir una pista de aterrizaje. Cuando poco después regresó, la choza había sido destrozada, faltaban los machetes y las ollas, y dos lanzas habían sido cruzadas frente a la puerta (Dall'Alba 1992, p. 100 ; Elliot 1989, p. 18). El dejar dos lanzas entrecruzadas - por lo general en sendas selváticas - era una costumbre huao que hablaba un lenguaje claro acerca de su disposición ante el contacto. Tidmarsh abandonó entonces el intento de hallar a los huaorani, pero sirvió de intermediario, enviando mensajes grabados entre Dayuma y la misionera Rachel Saint por un lado, que se hallaban en Estados Unidos, y dos mujeres huaorani que habían escapado recientemente de su grupo (Wallis 1996, p. 89). 


\section{Otras huellas de JoAQUina}

Además del documento aquí presentado existen unas pocas informaciones más sobre Joaquina. En 1947, llegó a la región el viajero y fotógrafo sueco Rolf Blomberg, que conoció allí a Mr. Souder, un norteamericano en cuya hacienda vivía la muchacha ${ }^{10}$. Éste le cuenta entonces que ella había vivido 14 meses con los huaorani, quienes la trataron muy bien, luego se casó y vive en la actualidad en la hacienda con su esposo. Souder hace la salvedad que la joven "se ha vuelto un tanto extraña » (Blomberg 1996, p. 25). Aunque no sabemos concretamente qué conducta le inspiró este comentario, evidentemente había diferencias en su carácter entre antes y después de su convivencia con los huaorani. Joaquina había presenciado el asesinato de la pareja con la cual viajaba el día del rapto y visto el botín ensangrentado que traían los huaorani de sus ataques al mundo exterior - que era su mundo. Verse obligada a vivir desnuda y haber sufrido el proceso de perforación de los lóbulos fueron tambíen sin duda experiencias traumáticas.

Poco tiempo después de regresar a la hacienda, Joaquina ofició de intérprete en las conversaciones con dos mujeres huaorani que habian sido capturadas y llevadas a un claustro en Quito, a fin de obtener de ellas informaciones para la posterior actividad misionera. Allí se realizaron algunas entrevistas, pero la enfermedad y la apatía se apoderaron de las mujeres, que murieron poco después (Blomberg 1996, pp. 47 ss.).

\section{LOS HUAORANI BAJO LA MIRADA DE JOAQUINA}

Si bien el relato de Joaquina pertenece al género autobiográfico, no se trata de una historia de vida en el sentido estricto del término, ya que ella no pudo elegir sus temas. La niña no cuenta espontáneamente sino guiada por las preguntas concretas de Tidmarsh. Algunas de sus observaciones parecen sin embargo inspiradas en una comparación de rasgos contrarios a los existentes en las sociedades quichua, como sus informaciones acerca de que los huaorani no castigan a las mujeres, no usan el alucinógeno floripondio (Datural Brugmansia sp.), no mastican la yuca para hacer chicha, no comen separados por sexos y que las mujeres se acuestan en una hamaca para el parto, en vez de dar a luz fuera de la vivienda (Tidmarsh \& Grefa 1945, p. 6 ; véase Rival 1998, p. 623). Es posible suponer lo que no mencionó a un extranjero adulto, misionero además : el hecho que los huaorani quisieron casarla recién un año más tarde se debió probablemente a que esperaron a que tuviera su primera menstruación.

La confiabilidad de las informaciones de Joaquina se ve reforzada por la presencia de dos cautivas quichua, que seguramente sirvieron de intermediarias en el conocimiento del mundo huao. La vida diaria transcurría armónicamente, no existían peleas entre los hombres, quienes tampoco pegaban a las mujeres. Su afirmación del buen trato recibido por parte de sus captores es tanto más confiable cuanto que no estaba dictada por la simpatía. Joaquina los odiaba, por lo cual no les indica que se debe cocinar el arroz, que habían obtenido en un pillaje, y se abstiene de intervenir cuando lo comen crudo. Su observación de la convivencia armónica de los huaorani no cuadra evidentemente con las opiniones y expectativas de los quichua, que le proporcionaron a Blomberg (1996, p. 31) como proveniente de Joaquina una versión lúgubre de la vida cotidiana, según la cual las mujeres viven en un miedo constante a los hombres. 
La composición del grupo con el cual vivió, su aprovechamiento del medio ambiente y su relación con el mundo exterior y las influencias surgidas por el contacto son algunos de los temas que se hallan en su relato.

La gente del grupo al que fue incorporada Joaquina eran llamados los gikitairi (véase Robarchek 1998, p. 98) y constaba de 34 personas, 11 hombres y 16 mujeres (sin contar a Joaquina, dos de las cuales eran cautivas quichua) y 6 niños. Este grupo residencial mantenía únicamente contacto con un grupo aliado que habitaba una vivienda situada a varias horas de marcha. Según Rival, los grupos residenciales obtienen un grado óptimo de endogamia y estabilidad autárquica a través de la hostilidad hacia los demás, que son enemigos. Cuando se da una falta de esposas, desacuerdos y divisiones internas, estos grupos se realinean en acuerdos con los anteriores enemigos, lo cual acarrea violencia y destrucción, que puede conducir a la desaparición de grupos enteros (Rival 1993, p. 367).

Dos informaciones del relato muestran que estos años estaban caracterizados por la paz interna y la guerra externa: los habitantes de la vivienda no apostaban centinelas y dormían tranquilos hasta el amanecer. En épocas de guerra interna, por el contrario, los huaorani levantaban barricadas alrededor de las casas, borraban sus huellas y apostaban centinelas (Yost 1981a, p. 111). Si un huao gritaba en broma que se acercaban los kowori (no-huaorani), la gente corría a esconderse en la selva - lo que revela al mismo tiempo el temor que estos inspiraban y también una de las formas del humor huao.

El cuadro que se desprende de las observaciones de Joaquina es el de un grupo que vivía libre de conflictos intraétnicos, en una paz interna que forma un fuerte contraste con la asiduidad de los ataques a los campamentos y a los trabajadores quichua. La experiencia de otra cautiva, Maruja, fue muy distinta, pues presenció numerosas peleas dentro de la misma vivienda (Cabodevilla 1994, p. 315). ¿Cómo se explican estas informaciones divergentes? Si bien Maruja vivió con el mismo grupo que Joaquina, esto fue en 1957, o sea, 12 años más tarde, de modo que sus diferentes experiencias reflejan respectivamente dos diferentes momentos de las cíclicas paz y guerra internas.

Cada pocos días los hombres emprendían expediciones de matanza, acompañados por jóvenes de ambos sexos. Los huaorani se preciaban de que los extranjeros no los podían matar, pero que ellos los estaban exterminando paulatinamente (Tidmarsh \& Grefa 1945, p. 6). Esta convicción se apoyaba seguramente en la concepción de que la sociedad oponente estaba compuesta por la cantidad de gente que veían. Los trabajadores de las haciendas y los campamentos constituían para los huaorani la totalidad del mundo conocido y se trataba de una cantidad de personas que confiaban poder exterminar. Si se tiene en cuenta que habitualmente un huao no veía en toda su vida más que unas $70 \mathrm{u} 80$ personas, la posterior toma de conciencia de la magnitud de estas poblaciones enemigas debe haber tenido consecuencias traumáticas, lo que se desprende aún hoy de las palabras de los ancianos ${ }^{11}$. Esto puede haber dado paso a la creciente convicción que nunca llegarían a exterminarlos.

En lo que hace al aprovechamiento del medio ambiente y las actividades de subsistencia, las informaciones de Joaquina muestran, por una parte, aquello que la impresiona por su diferencia con respecto a las costumbres quichua, por ejemplo, que los huaorani no cazan ni comen danta (= tapir, véase también Rival 1996, p. 210). La fundamentación de la no-caza del tapir en una insuficiencia tecnológica, ya que no se 
los podía cazar con lanza (Robarchek 1998, p. 108), no tiene asidero en la realidad, pues tanto los huaorani (Yost \& Kelley 1983) como otros grupos indígenas los cazan o cazaban exitosamente con lanzas.

Para la observación de Joaquina de que las mujeres utilizaban lanzas para cazar pecaríes, no encuentro ratificación en la bibliografía : en la actualidad algunas mujeres participan a veces de la cacería, para lo cual utilizan las cerbatanas de sus padres o hermanos (Rival 1996, p. 207).

Es muy curiosa la información de que la esposa del jefe de aquel entonces había obtenido hacía mucho tiempo yuca y plátanos, que comenzaron por tanto a cultivar (Tidmarsh \& Grefa 1945, p. 6). Esto podría remontarnos a alrededor de 1910, pero una adquisición tan tardía de estos cultígenos no es verosímil. Quizás esta aseveración refleje un pasado de guerra interna, en el cual este grupo tuvo que vivir escondido y perdió el acceso a sus chacras. De todos modos, la yuca no era indispensable para la supervivencia, pues los huaorani podían prescindir de ella muchos meses y alimentarse del producto de la caza y la recolección (Rival 1996, p. 211).

Las informaciones difieren con respecto al procesamiento de los tubérculos de yuca para hacer bebida: Joaquina observó que (a diferencia de los quichua) las mujeres no masticaban los tubérculos, mientras que 30 años mas tarde, Yost (1981a, p. 108) observó que la procesaban de igual modo que los quichua. Cabe preguntarse si éste fué un rasgo divergente entre grupos, o si fueron las cautivas quichua quienes transmitieron la técnica de la masticación.

En cuanto al aprovechamiento de los recursos naturales en la alimentación, los huaorani utilizaban por lo menos dos frutos (un tubérculo dulce y un fruto rojo arbóreo, Tidmarsh \& Grefa 1945, pp. 6 ss.), que los quichua no conocen. En un estudio etnobotánico, Cerón y Montalvo (1998, p. 195) señalan que numerosas especies utilizadas por los huaorani como alimentos no han sido registradas como tales para otros grupos de la región, aunque lamentablemente no las identifican. A su vez, los huaorani no aprovechaban frutos de uso general en el área, ya que Joaquina informa que consideraban venenosa a la papaya. A pesar de ser ágiles trepadores de árboles, tampoco recolectaban miel.

Joaquina no presenció la pesca con barbasco, que sí se practica en la actualidad, aunque esto no permite concluir que se trate de una adquisición reciente. Los huaorani utilizan con este fin siete especies botánicas diferentes (Cerón \& Montalvo 1998 , p. 228), una diversificación que habla probablemente a favor de la antigüedad de la práctica. La utilización se halla documentada además desde la generación de los abuelos de personas que hoy son ancianas (Wallis 1996, p. 48). Dado que durante la estadía de Joaquina, el grupo estaba fuertemente orientado a la observación del movimiento de los intrusos, a los pillajes y a la guerra, quizás estas actividades tenían preeminencia sobre las otras.

La confección de hamacas era una tarea tanto femenina como masculina. Alrededor de 1970 era en algunos grupos tarea de los hombres, en otros de las mujeres (Yost 1981a, p. 109). De los ejemplos anteriores se deduce la imposibilidad de generalizar o hacer normas exclusivas de las costumbres huao, imposibilidad que ha sido comentada certeramente por Cabodevilla $(1994$, p. 215) : « estas pequeñas sociedades aisladas pasaban por tantos incidentes y transformaciones violentas como para verse obligadas a no pocas excepciones $»$. 
El pillaje y la actitud contestataria frente a la colonización en la región habían determinado ya alrededor de 1944 un cambio de territorio y la adopción de nuevos elementos. Una de las cautivas (Margarita) afirma que los huaorani se habían mudado más cerca de los asentamientos para acceder más fácilmente a las herramientas, pues no cultivaban lo suficiente (Blomberg 1996, p. 33). Si bien formaban una sociedad netamente interfluvial, tenían algunas canoas obtenidas en sus incursiones, aunque ignoramos si las utilizaban.

Los huaorani valoraban por su practicidad algunos objetos obtenidos en los pillajes y los utilizaban en la vida cotidiana, como fósforos, hachas, machetes y cuchillos. Sin embargo no utilizaban otros objetos, como sartenes y jofainas, que evidentemente conservaban como trofeos; en las palabras de Tidmarsh, « as mementos of their victories » (Tidmarsh \& Grefa 1945, p. 4). Es de destacar que, desde nuestro punto de vista, todos los objetos mencionados son igualmente prácticos, pero no lo eran para los huaorani de entonces. Las ropas que obtenían en los pillajes poseían igualmente la condición de «trofeo », pues se usaban para la fiesta que se realizaba luego del regreso de los guerreros y se guardaban para ser utilizadas en la próxima ocasión. El hecho que para realizar un casamiento se vistieran con las ropas de los enemigos (Tidmarsh \& Grefa 1945, pp. 2, 6) muestra que éstas habían alcanzado un alto valor simbólico. En esa época, las ocasiones festivas eran numerosas, ya que, además de las tradicionales, como el casamiento, se festejaba el regreso de cada expedición exitosa, y, como veremos a continuación, cuando lograron « herir » al avión.

Para establecer el contacto, la táctica de los misioneros (y luego también de las compañias petroleras) fue dejar caer objetos desde helicópteros o aviones. De la expedición de los cuatro misioneros masacrados en 1956 por los huaorani se recuperaron las cámaras fotográficas encontradas junto a los cadáveres. Entre las fotos que pudieron ser reveladas, una muestra a un huao con una hamburguesa en la mano, acompañada de la leyenda : "enjoys his first hamburger » (Elliot 1981, p. 117).

Las mujeres dedicaban gran parte del día a observar el movimiento de aviones en el campamento de Shell y explicaron a los hombres que era un hombre-pájaro y que se lo podía matar. Una vez que un avión dejó caer regalos, se interpretó como muestra que lo habían herido con sus lanzas y obligado a que dejara caer los objetos que se hallaban en su interior ${ }^{12}$. Este acontecimiento dio lugar a una gran fiesta (Tidmarsh \& Grefa 1945, p. 3 ; véase también Eliott 1981, p. 135). Para apresar y matar al extraño animal, los hombres talaron un gran árbol, el cual erigieron cerca de la casa, a fin de provocar una colisión. El hecho que el avión sobrevolara a gran altura les provocó una gran frustración.

A la luz de estas informaciones, es evidente que la interpretación de los huaorani acerca de los « regalos » era antipódica a la intención de los misioneros y petroleros. Mientras que éstos pensaban que así mostraban su deseo de establecer un contacto pacífico, para los huaorani estos objetos significaban una victoria, pues habían herido al ave y lo habían obligado a arrojar el contenido de su estómago. Cuando los objetos que caían desde el aire no alcanzaban para todos, las discusiones conducían a veces a homicidios (Blomberg 1996, p. 50).

Como ya ha señalado Yost (1981b, p. 688), en algunas áreas huao se dieron rasgos que recuerdan a los cargo-cult de Oceania. Alrededor de 1970, los huaorani realizaban 
rituales que imitaban algunas actividades de los campos petroleros y cantaban canciones en las cuales los protagonistas eran helicópteros. El ritual acompañado por el baile tenía como fin atraerlos para que arrojaran desde el aire hachas, machetes, ropa y ollas de aluminio. Incluso un grupo alejado construyó por propia voluntad una pista de aterrizaje para que los aviones pudieran traerles regalos (Yost 1981b, p. 688).

La actitud huao con respecto a los aviones y los regalos muestra un cambio con el correr del tiempo : en los años 40 se veía a los aviones como seres animados que podían ser heridos y a los cuales trataban de vencer. Cuando la misión protestante afianzó su influencia - época de la que datan las observaciones de Yost - la concepción huao sufrió un cambio : los helicópteros y aviones ya no eran seres vivientes a los que podían herir, sino seres cargados de codiciados objetos, a los que buscaban atraer por medio de cantos y rituales.

En el relato de Joaquina hay pocos indicios de las creencias religiosas huao, por lo demás, un aspecto hasta hoy poco conocido.

\section{LAS CAUTIVAS EN OTRAS SOCIEDADES}

Una comparación breve entre la toma de cautivos entre los huaorani y otras (muy distintas) sociedades indígenas, permite ver más claramente las modalidades de este fenómeno entre aquéllos. El caso más notable, tanto por el alto número de cautivos como por la continuidad de esta práctica, fue, a partir del siglo XVIII hasta las postrimerías del XIX, el de las sociedades de Pampa y Patagonia (pampa, tehuelche, mapuche) en Chile y Argentina. Los cautivos (generalmente mujeres) eran capturados en el curso de pillajes a viajeros y a establecimientos agrícolas. Tanto su procedencia étnica como su modo de incorporación a las sociedades indígenas fueron variadas. Las cautivas eran por lo general criollas pero también españolas y francesas, que se convirtieron en esposas o concubinas de indígenas, pero también fueron nodrizas, secretarias y traductoras en las transacciones y tratados de paz con las autoridades. Tenían también valor como rehenes, pues eran vendidas, trocadas e incluso formaban parte de la dote que aportaban al matrimonio personas de familias destacadas. De ser rescatadas, su destino posterior era a menudo de vergüenza y ocultación, pues en la sociedad colonial significaban un deshonor para sus familias, razón por la cual no pocas mujeres prefirieron permanecer junto a sus hijos en la sociedad indígena (Altube 1999, Sosa 2001 ; Lázaro Avila 1994 ha analizado estos casos para los siglos XVI y XVII en Chile) ${ }^{13}$. La vergonzante forma de reinserción de las mujeres rescatadas explica en parte que existan relatos de cautiverio de hombres (véase por ej. Avendaño en Hux 1999, Avendaño 2000), pero aparentemente sólo uno de una mujer, recogido por Carbajal (1995), aunque el número de cautivas haya sido mayor. Si bien los ex-cautivos de uno u otro sexo eran mirados con desconfianza por haber convivido con los « salvajes », en el caso de mujeres se sumaban los prejuicios típicos de género : habían sido violadas y tenido hijos mestizos. Por su parte, las mujeres y niños indígenas tomados prisioneros por las autoridades fueron deportados o entregados en " adopción » - un eufemismo para denominar su condición de empleadas de servicio doméstico.

Tanto entre estas sociedades ecuestres como entre los huaorani, los pillajes y ataques eran estrategias de la guerra. Las características señaladas como formas de la 
guerra en Pampa y Patagonia (ataques sorpresivos, arreo de ganado, rapto de mujeres, muerte, regreso veloz a sus territorios, Crivelli 1997, p. 180) son iguales a las de los huaorani, si suplantamos el arreo de ganado por el pillaje. Las diferencias entre ambos fenómenos radica en las diferencias de estas sociedades entre sí : las de Pampa y Patagonia eran sociedades estratificadas, en las que existía una especie de nobleza, y sus relaciones con la sociedad exterior eran más habituales. Esto determinó también el papel distinto que desempeñaron aquí las cautivas, que eran vendidas o canjeadas, mientras que los huaorani las incorporaban a su sociedad.

\section{CONClusiones}

Las informaciones de Joaquina, que se basan en sus experiencias de 1944-1945, conforman un documento excepcional, ya que, por lo general, la adquisición de conocimientos acerca de una sociedad indígena presupone algún tipo de contacto pacífico. En el caso de los huaorani, los datos más tempranos provenían hasta ahora de la época de su pacificación, o sea alrededor de 1960.

El hecho de haber hallado este documento después de haber abandonado la región me impidió tratar de localizar a Joaquina y a otras antiguas cautivas allá, lo que intentaré en un próximo viaje. Dado que eran muy jóvenes en 1940-1950, varias de ellas deben vivir todavía ${ }^{14}$. El objetivo de estas entrevistas no radicaría tanto en recabar información que a Tidmarsh podría habérsele pasado por alto, sino sobre todo el de conocer su punto de vista actual sobre su época de cautiverio, cuando, después de tantos años de conflicto, las relaciones con los huaorani son más habituales y éstos han perdido en parte para los quichua la connotación de horror que tenían en épocas pasadas.

El documento corrige una idea que se ha generalizado con respecto a esta sociedad : la de su aislamiento durante siglos, tanto biológica como culturalmente. A la luz de este documento, la afirmación de Rival (1996b, p. 146) acerca de su « aislamiento drástico " y la ausencia total de rasgos no-huaorani hasta los años 60 , cuando se dio el primer contacto, debe ser afinada. Como lo vimos, los huaorani utilizaban distintos objetos obtenidos por pillaje (fósforos, cuchillos, machetes) y poseían algunas canoas. Incluso conservaban ciertos objetos por su calidad de trofeos, y el uso de la ropa occidental había adquirido un valor ideológico y simbólico.

En cuanto al aislamiento biológico, por el cual la mezcla entre huaorani e individuos de distinta filiación étnica habría sido nula, la existencia de cautivas entre 1940-1965, muestra que aquél fue parcial. Aunque ignoramos el número total de cautivas que vivían en esa época en los diferentes grupos residenciales, en el caso tratado aquí, era considerable : de un total de 16 mujeres, 3 eran cautivas, y 2 de ellas tuvieron hijos con huaorani. Si bien la cantidad de cautivas existente en este grupo residencial no tiene por qué ser extrapolable a otros, muestra que no es adecuado partir de la suposición de un aislamiento absoluto.

Anteriormente al informe aquí tratado, la información sobre los pillajes y ataques huaorani procedía de los trabajadores de las haciendas y las compañías petroleras, pero nada se sabía de la visión de los huaorani acerca de aquéllos, ni de los rituales y festejos a que daban lugar, ni tampoco de la conservación de objetos ganados a los enemigos en calidad de trofeos. Este último comportamiento evidencia hasta qué 
punto los huaorani vivían los conflictos con el frente de colonización como una guerra.

Las formas de la guerra huao presentan varios puntos en común con las de los yanomami de Venezuela investigadas por Ferguson (1999), como la relocación de asentamientos a fin de hallarse más cerca de las fuentes proveedoras de objetos de metal, el pillaje como forma de acceso a las manufacturas occidentales y el surgimiento de conflictos políticos (Ferguson 1999, pp. 206, 216 s.). En el caso de los huaorani se agregaba una fuente más de conflicto, pues el hecho que los objetos se dejaran caer desde el aire hacía que su obtención fuera a menudo meramente casual, lo que originaba disputas internas que conducían incluso a homicidios.

Partiendo de las informaciones de Joaquina, es posible plantear una hipótesis con respecto a los motivos de pacificación con el mundo exterior que tuvo lugar alrededor de 1960. Las explicaciones para la pax huao que se encuentran en la bibliografía dependen de la posición de sus autores : para los misioneros protestantes la pacificación se debió a haberles llevado la palabra de Dios. Los Robarchek (1998) argumentan desde distintos ángulos, lo principal sería la decisión de los mismos huaorani.

Creo que es posible hacer otra lectura de la pacificación : en 1944 y durante los años siguientes, el incipiente frente de penetración estaba compuesto de pocos individuos. Las 10 ó 20 personas que los huaorani observaban y atacaban no hacían mella en su confianza de que podrían vencerlos algún día. Pero con el tiempo, los signos funestos aumentaron: el creciente número de aviones que surcaban el cielo, las mujeres del grupo que huyeron hacia el exterior y luego regresaron y, sobre todo, lo relatado por Dayuma sobre su estadía en Quito y en los Estados Unidos, les hizo conocer en un tiempo relativamente breve la vastedad del mundo de los otros, y que el número de éstos era inconmensurable. De ahí a la aceptación de los guerreros que nunca lograrían vencerlos, el paso fue breve, de modo que la pacificación huao fue en parte producto de la toma de conciencia de la magnitud de las poblaciones enemigas y de la convicción de haber sido vencidos.*

* Manuscrit reçu en avril 2002, accepté pour publication en juin 2002.

\section{Unarrangeid Notes on the Aucas of the Nushino Basin Miscellaneous Unarranged Notes on the Aucas of Eastern Ecuador}

[1] ${ }^{15}$ Early in May 1945, Joaquina Grifa, a yumba or Quichua speaking Forest Indian girl escaped from the Aucas who had taken her captive just over a year ago as she was going up the river Arajuno with other Indians in a dug-out canoe ${ }^{16}$.

She gave the following information of the aucas, their customs and language. Most of the details were obtained directly from her, but some were obtained through Srta Ella Souder ${ }^{17}$.

Estimate of number of aucas varied in different accounts, and at various times suggested that there were perhaps thirty men, twenty men in each of two houses (communal), seven families in a house. A normal family consists of husband, two wives, four or five children by first wife, one or two by second. Hence assuming twenty male adults, the number of aucas would be in the neighbourhood of a hundred and eighty to two hundred including all children.

Two main auca houses, one near Shell camp, other nearer River Napo in the region of the river Sumino or perhaps south of the Nushino in that region. One of the Curagas from time to time uses a house across the river Sapino (or Tsapino). 
When she was captured, a man caught her by both hands and swung her over his shoulders and carried her into the forests, later, he made her walk and led her by the hand. The band of aucas followed the 'barradero' [sic, varadero] ${ }^{18}$ from the lower Arajuno, across the Sotano and the Nushino, and onwards in the same direction. She was captured about nine in the morning, and they reached their house before nightfall. She was given a hammock, but threw it into the river, the Auca Yacu. She seems to have been well treated, and lived and was treated like all the other women. They would go out to the chacras at early dawn, and continue working till darkness without returning to the houses during the day when it was really fine weather. Toward the end of her time with the aucas, they wanted to marry her, but she refused ; and the women told her one day that on the following day there would be a great feast, and if she would not consent to be married, she would be killed ${ }^{19}$. Consequently, taking the advantage of the moonlight, she fled from the house when all were asleep (she says in the middle of the night, but to an Indian that could signify any time after say 9 p. m.), and knowing the direction in which the Napo lay, she escaped through the forests, and arrived on the banks of the Napo river in the neighbourhood of Sunino Chicta at about dawn. Shortly, a canoe came by, and a man wanted to shoot her, but an old Indian restrained him, saying it was but a girl. She called out to them to take her across the river, and was able to explain that she had been captured by tha aucas, and had escaped. After that she lapsed into auca language, probably overcome by the nervous strain of her ordeal. These Indians took the girl to Santa Rosa and gave her clothes. Later Mr. Souder hearing of her escape sent and took her back to his home.

The aucas take a great pride in their hair. It is long and abundant, and they encourage growth by treatment with concoctions from forest plants.

All sleep in hammocks, men and women in different parts of the house. The hammocks are strung up in lines, the height above ther [the] ground varying with the size of the hammock and its occupant. A fire burns under each hammock. Little boys sleep with the father [2] little girls sleep in the same hammock with their mother until big enough to have their own hammock. Men make their own hammocks and women make the women's hammocks.

The women who go out to work all day in the chacras come back heavily loaded with food which they divide with the women who did not go back but were occupied with duties in the houses.

The aucas live in perfect harmony. She never saw men fight, or saw a man strike a woman.

The men have two wives, the first may have four or more children, but the second is put to death apparently with great festivity after giving birth to a second child. Joaquina insists that such a man does not marry a second wife, but this seems improbable. Often the two wives are sisters, and often the second wife is taken as a little child and lives with her future husband until she is old enough to be married. The second wife is killed with lances, and seemingly with great rejoicing ${ }^{20}$.

When the men return from a killing expedition, having caught or killed victims, there is great rejoicing. The men drink much chicha, then retire to their hammocks. The women then beat them with nettles (ortiga) until they fall asleep. These victories are celebrated by a great feast in which they have a dance. In the dance the women gather into two groups in the centre, all holding hands, and the two groups march toward and away from each other in rhythm; at the same time around this group the men jump and leap brandishing their lances. At these feasts all put on clothes that they have captured, regardless of sex, thus the men may array themselves in trousers or with skirts. On their heads they put crowns of vertically placed long white feathers of the garza, and on the crown or back of the head, they put the dried skin and brilliant blue plumage of the lushan pishcu ${ }^{21}$. These adornments are thrown away after the dance as they become damaged, but the clothing is guarded in zaparos until the next occasion.

The aucas know how to make saparos, double woven baskets with leaves placed between the two layers of weave, overlapping in such a fashion that water falling from above will not enter. On these occasions the men bore hores [holes] through the nose just above the alae nasae and put 
small balsa sticks through horizontally. The holes are allowed to close up after the feast until the next occasion.

Men and women alike wear thick balsa sticks through holes in the lobes of their ears.

When the men return from an expedition without having taken or killed any victims, they are furious, and the women often run away and hide in the forests.

[3] On one occasion, Joaquinta [sic] was taken by the auca women to watch the aeroplanes at the Shell Camp on the river Arajuno. She heard the women discussing as they saw the planes come, be unloaded and return to their base camp. One said : It is a man ; it MUST be a man ; whereupon the girl explained that it was a kind of « house built like a canoe » bringing things to the camp, for she remembered seeing the amphibian plane coming to the lower Arajuno when she was free. But the women would not believe this and declared this strange creature a " man-bird » - for did it not have ist arms stretched out on either side ? But, if it is a man, they reasoned, it must be woundable, and so they could kill it. So this decision was communicated to the warlike menfolk who have a terrible thurst for human blood, and they started throwing spear up at the plane whenever it came over their houses. But as the lances could not reach the 'plane' they decided to bring the tallest tree in the forests and erect it near their house so that the man bird would strike against it and fall, and then they could truly kill it. And they were furious when on the next visit from the air, the plane passed high above the clouds. A little later, round about last Christmas a plane dropped some beads, clothing and other objects over the auca house. These were eagerly picked up with the explanation : Look, it is wounded, these have dropped out of its « inside ». And so a feast and dance were held to celebrate the wounding of the man-bird.

Joaquina never saw an auca drunk; they take unfermented chicha made from boiled yuca (manioc) which is not masticated by the women as is the custom among other tribes of Indians.

She noted perfect harmony among the aucas. The men never fought, and one was never seen to strike a woman. Only when the men returned from an unsuccessful foray did the women hide, and that from the passing fury of the disappointed warriors.

The aucas have plenty of matches which they use now instead of their little fire sticks which they formerly rubbed between their hands on a block of hard wood.

They have money, believed robbed from Indian houses at Achupara on the Napo river.

They have plenty of axes, machetes, and knives which they use. All these are spoils from robbing raids. They also make and use scrapers made from fresh-water shells that they sharpen by rubbing on stones. And they make knives from the hard wood of the chonta and related palms. These they use mainly for culinary purposes, such as the peeling of the fruit of the chonta palm.

Aucas know how to swim, and thus is disposed of an old belief that they cannot swim or cross larger rivers.

In making chonta spears, they formerly scraped the wood with shells, but now use knives.

They make and use blowpipes.

She saw no gold among them.

The aucas divided the strings of beads each man taking a single string.

The roofs of the aucas come down nearly to the ground, and their doors are very low.

They do not make canoes, but have three stolen ones taken from the river Nushino.

They have no hens, and she saw no eggs of any kind, and could give no word for egg.

[4] Joaquina could tell but little about the aucas mode of counting, saying that they use their fingers and perhaps toes. A few numbers that she gave were quichua, and all disarranged thus she gave three for one, and four for two.

From the river Arajuno to the auca house was about nine hours walk.

The aucas have saucepans (kettles), basins and other objects stolen from houses, and these are not used, but guarded as mementos of their victories.

The aucas named the aeroplane 'wina', and thought to wound its abdomen with their lances. 
Among the aucas are two other yumba women, robbed from the Napo (? Achupara), and perhaps one from Huitu (?? $)^{22}$, named Margarita and Victoria. These have now become incorporated in the auca tribe and are married to auca men.

On one occasion the auca women took Joaquina with them to the banks of the Napo river near Santa Rosa where hidden in the undergrowth, they watched the canoes passing. This helped her to know in which direction to travel when she ran away.

J. said that among the aucas were no flies, mosquitos.

The aucas visited the house and neighbourhood of an Indian, Shanri, near Achupara.

She played with the auca young women when they would bathe together in the river.

The Indians do not seem to poison the rivers, and do not have barbasco.

She said that the Indians have no witch-doctors, only two chiefs, and was unable to give me a word for God, demon, or spirit ; while among the yumbos the latter two are very strong concepts.

She could tell but little about the legends and stories of the old people, who talk much among themselves but did not speak much to her.

They have no hearthstones; pots are put directly on to the fires. They use just any of the hammock fires for cooking, and do not have a special fire for this purpose. Near the fire they have a very small representative of the other Indians 'mantaca' or shelf for drying meat, etc. The aucas talk of stone machetes, but $\mathbf{J}$. did not see any ; [varias palabras tachadas] ${ }^{23}$

The women tell that in the " olden times " they had no yuca or platano, and lived mainly on fruits from the forest and game, and fish ; but one day the wife of the present chief or " curaga " went on a very long journey, and brought back with her platanos and guineos, and yuca, which from that time onward they cultivated and adopted as foods.

Aucas tell how they visited big house on the Arajuno, and heard the occupant snoring, and wanted to kill him, but had left their lances on the other side of the river. (This would seem to have been Dr Tidmarsh's house as he had no dogs, whereas Mr. Sou- [Souder] kept dogs, and had many Indians living around him ; moreover, the river Arajuno would be diflicult to cross near Souder's and at night.) Further, the auca path led down to the Cusano river just where the path from Dr Tidmarsh's house goes to that river:

Joaquina saw wild honey, but the aucas don't eat it.

[5] Joaquina tells that two women made « strong », i.e. fermented chicha, and the auca men killed them in punishment.

The aucas don't seem to find or eat any eggs.

They weave hammocks, and " ishingas » - small round nets for catching fish, but they do not make the very similar shigras, or carrying nets, (bags), nor do they make « llicas » or long weighted nets for throwing into the shallows, and they have no « atarayas » - big round nets for throwing. They do have and seem to use stolen shigras.

Joaquin [sic] aflirms that NO AUCA MAN SPEAKS QUICHUA.

The aucas tell that they have visited Indian houses at night and entered them and listened to the Indians, all of whom were drunk. Aucas say they heard the word « amonrahuai », but this is not a form used by the Indians although the ending is a very common one.

The women in cooking yuca stand the tubers vertically in the pots of earthenware.

They use knives of chonta for preparing yuca.

Joaquina asserted that there was no illness among the aucas ; and when pressed declared that she saw no fever or dysentry, nor heard words for these. She told however that a man had had an illness 'like a fit' and had died. An auca who was pierced by a ray (fresh-water skate), poured very hot water over the wound. She could tell nothing of medicinal herbs in use among them, and repeated that they do not have illnesses.

They catch fish with small lances as well as with nets. 
Women as well as men use lances to spear wild pigs, the women using a smaller size than the men.

There were no bows and arrows, but the Indians make their own blowpipes.

When she reached the auca camp after capture, the aucas gave Joaquina a hammock, but in temper she threw it away in the river, but later they gave her another one the same night.

After a few days, they took away her clothes. She pleaded to be left with her skirt, but they took that away too. They bored her ears for the balsa sticks, and wanted to pierce the nose too but she refused.

She did not recognise any of the words previously published as being of auca language, but which are of unmistakeable Zaparo type. Also the auca word for platano is quite distinct from the word for platano in the Shiripuno language ; and the Aushiri vocabulary published in [el autor deja aquí un espacio, probablemente para completarlo más tarde] ${ }^{24}$ is quite distinct ; hence it would seem that this is a little tribe hitherto unrecognised as being a separate entity, with a hitherto unheard language, and of unknown tribal customs and relations until these data were brought out by Joaquina Grifa.

She insists that they do not go on long treks toward " the rising of the sun "; and that they have no connection with any other groups of Indians. Joaquina told how that three days before she escaped, the aucas went out and raided the Shell house at El Capricho. The men returned bringing machetes, blankets, and a tin full of rice [6] with the blood of one of the three victims. They did not know how to prepare the rice, and Joaquina would not tell them because of her hatred of them. So they thought it must be prepared like chicha, and mixed the uncooked rice with water and drank it !

The aucas do not defecate into the rivers and streams as do the yumbos, but squat on a section of tree trunk. Men and women alike follow this custom.

The women make the earthenware pots by the same method as theyumbas, but they burn them with any dry wood available and not with bamboo wood alone as is the custom of the yumbos.

When the children cry, they wail : Baráa. baráa, baráa, which is the equivalent of mother, mother, mother.

The aucas eat raw a sweet tumber that she did not recognise. (It looks like white camote, white sweet potato).

Much camote grow wild in the river banks, and the Indians cook and eat it.

They make feasts for the lads at puberty, which consists in taking chicha made by a little girl ; the whole group drink and then they dance all the night. She could tell of no excursion by the youth into the forest under the influence of " wanduj » a variety of floripondia. There is much floripondia on every hand, but the aucas do not use it. Women give birth to their children in hammocks, and not in accordance with the custom of the yumbas. At childbirth, both parents go to bed, and fast from certain foods, especially from the meat of deer and wild pig, although the woman will partake of these up till the day she gives birth to the child.

The aucas, usually all the men, and the youths (male and female) go out on expeditions every few days in search for victims (or " paja » as they say). They are away two and three days at times, and in such case take food with them.

Aucas say that the " cuguris » or outsiders have not killed any of them, but they are gradually wiping out other races.

Aucas say that they came from the Curaray.

They seem to have no form of greeting, and the men enter into the houses from a journey silently and say nothing.

In the morning, the women get up first and eat food prepared the previous day ; then when the men see the women eating they come and join them. They eat in family groups, and not in two groups, one of males, and the other of females as do the yumbos and the Colorados.

The aucas make no traps (tictas, panwas, etc). They do not eat the flesh of dantas which abound in that region. 
Wedding ceremony [is] very simple. Youth embaraces the girl of his choice in front of a pot of chicha, and then they have a typical dance arrayed in their stolen clothes.

The main food is yuca, but they do make « chucula » out of ripe platanos by boiling and then crushing in the water. They apparently eat green platanos raw.

They do not have the small flute which the yumbos make out of bones - known as " pingullus », but they do make a short flute out of bamboo stem. Women make tasas, but they do not seem to know how to make the open weave " ashangas ».

The women appear not to take haemostatic herbs at menstruation or childbirth as is custom among the yumbas.

Aucas don't eat frogs, and they have no potatos.

[7] The aucas eat a fruit which they call « umaya », red and growing on large tall trees. Joaquina could not tell us its name in Quichua altho' she seemed to believe that it does occur in the forests of the Arajuno.

There are rather wide variations in the colour skin of the aucas, some being lighter, and other darker than the usual color of the Quichua Indians.

The men whistle with the aid of their hands.

When the aucas dance they first cross their legs and stamp with their feet. The men dance first, and then the women.

Joaquina could give no information as to the auca beliefs concerning the dead.

As they dance they sing something like " ee ee ee pinun ".

The aucas tell how that once when they were travelling near the Napo having with them a young yumba woman, the latter heard voices of her own race and called out for help. Whereupon the Indians attacked the aucas and drove them to flight, and advanced to the auca home and robbed it.

Joaquina speaks of a big black (? burned) log that lies on the beach near the aucas house, which they call Tiniwanka, and which according to their beliefs came down from heaven. But she seemed very confused when she first spoke of it. Later on being asked again what tiniwanka was, she exclaimed : Wanka sent it ; it fell down from Wanka. Possibly this word is the name of the Deity.

Later on asking further about dead, she said that they make a sweeping upward movement of the arm and say " Phuu » to indicate the departure of the soul.

In order to carry the children, the women make a narrow cloth from the bark of the lanchama tree, and use it much as the yumba women do.

They keep no watch at night, and sleep without care especially just before dawn. At dawn they arise. The children are very alert and give warning at any unrecognised sound. Sometimes in play someone shouts out : The cuguris are coming, and then all flee and hide in the forests.

The aucas have no lights at night, and do not use copal.

They often paint themselves white with a white clay found not far from their houses. They also paint themselves with black wavy lines, round face, body and legs.

The men seldom go out unarmed, if ever. They usually take an armful of lances over their shoulder, at least ten apiece.

Both men and women go out at night, but usually return back before the small hours of the morning.

They do not grow sapallos.

They have no tobacco, and do not smoke.

In the chonda season, they make chicha from its fruit.

They do not make " cato », i.e. meat soup with grated platano, and they put only yuca in their soups.

They have no salt, and Joaquina mentioned no substitute.

She says that they have no seats or stools in their houses, and that the aucas converse standing, the men with men, and the women with women in groups apart. 
They believe papaya and the caracol or tree snail to be poisonous.

She noted no bats.

The women do not masticate the yuca to make chicha.

The aucas told that they had heard the yumbos shout : Aucas auca, when they made attacks.

Names of the aucas.

They appear to have but one name.

Men

Curaga at Nushino... Wamari

Curaga at Tsapino... Muipa

Gustavo [nota 1 de Tidmarsh : It is interesting to note this Spanish name, and arouses a considerably query as to its origin]

Wawa [nota 2 de Tidmarsh : A quichua word] ${ }^{25}$, Namu, Warkama, Kikita, Gava, Chantoa, Kara, Umara

\section{Women}

Aliva... curaga's wife

Api (Victoria), Balmira or Yumatika (Margarita), Mintaka ${ }^{26}$, Maikama, Maigari, Dayuma, Akao, Ersila (Joaquina), Lita, Boganai, Umatiki, Umiga, Ganwa, Miyima, Gami or Ngami, Umi

Children

Boys Zhuvi (chief's son). Wira, Kimu, Numa, Nampa ${ }^{27}$

Girl Nahua

\section{NOTAS}

1. Agradecimientos : la Deutsche Forschungsgemeinschaft (Sociedad Alemana de Investigación) financió mi estadia de siete semanas en Ecuador en el inicio de un proyecto sobre historias de vida huaorani. En Quito, Stephen Beckerman fue generoso como anfitrión y al compartir sus informaciones de campo. Trabajar en el Archivo histórico del Banco Central (Quito) fue un placer gracias a la amabilidad y eficiencia de Honorio Granja. Eva König, del Museo de antropología de Hamburgo, me proporcionó copia de fotos de los auishiri, tomadas por los fotógrafos Charles Kroehle y Georg Hübner en los años 1888-1889. Eduardo Crivelli me hizo conocer el relato de Carbajal (1995).

2. En la actualidad esta concepción ha perdido vigencia, pero la desconfianza hacia los extraños sigue incólumne : en 1990, corrian rumores que los ingenieros petroleros habían asesinado a varios huaorani, a fin de enlatar su carne para ser utilizada como comida para perros y gatos (Rival 1996, p. 72).

3. Posiblemente era Api la empleada en la casa de Quito de Carlos Sevilla, el dueño de la hacienda Illa, de quien dos misioneras del ILV intentan aprender la lengua huao. Esto no fue posible, ya que Api entremezclaba palabras quichua, que Sevilla atribuye a que es una quichua que vivió muchos años con los huaorani (Wallis 1996, p. 30).

4. Por ejemplo los secoya del río Aguarico, cuya tradición oral es en muchos aspectos confiable desde el punto de vista histórico, diferencian claramente a los auishiri de los « auca ». A su vez, los huaorani con los que hablé a principios del 2001 negaron terminantemente que los atributos que aparecen en las fotos de los auishiri tomadas en 1888-1889 por los fotógrafos alemanes Hübner y Kröhler (conservadas en el Museo de antropología de Hamburgo) sean huaorani.

5. Una viva muestra de esto es el informe escrito en 1742 por el jesuita Magnin (véase Cipolletti 1998).

6. El autor del documento escribe " Grifa ». Me he decidido a hacer esta corrección, pues « Grefa » es la forma habitual de este apellido en la región.

7. En un proyecto sobre historias de vida huaorani, viajé en enero del 2001 del territorio huao a Quito con la intención de regresar una semana más tarde para lograr traducciones más fieles de lo grabado. Una sublevación indígena bloqueó las rutas durante dos semanas, las que empleé en rastrear en los archivos material sobre el enigmático pasado de los huaorani. 
8. Además del informe de Tidmarsh existió evidentemente otro informe sobre el cautiverio de Joaquina, escrito por David Cooper, un joven misionero norteamericano, jefe de la estación misionera de Archidona, y que es el citado por Blomberg (1996, p. 31).

9. Dado que los misioneros protestantes querían ser los primeros en contactar a los huaorani, el silencio de Tidmarsh puede haber tenido por fin impedir que otros grupos (como los josefinos) pudieran valerse de sus informaciones. El punto de vista canónico sobre la actuación de la misión protestante en la región lo presentan escritos de miembros del ILV, como Elliot 1981, 1989 y Wallis 1996. Los trabajos críticos son numerosos, entre otros, Stoll (1992). Una visión a la vez crítica y mesurada es la de Cabodevilla (1994, p. 341 ss.).

10. Aparentemente nunca intentó comercializar los productos de su hacienda. Como la mayoría de los destinos de los pioneros en esa región y en esa época, el de Sam Souder posee numerosos ribetes novelescos (véase Cabodevilla 1994, p. 259).

11. Ome (=Umi), que huyó en 1947 de su grupo junto con Dayuma para escapar a las matanzas internas, quedó paralizada por la sorpresa y el terror al ver la enorme cantidad de gente que vivía en un pueblo cercano. Según sus palabras, los huaorani pensaban que las hormigas estaban compuestas por muchos individuos, pero jamás los seres humanos (Ome, comunicación personal, Toñampare, enero 2001).

12. Es probable que, dado que se trataba de un ser vivo, los huaorani concibieran que los objetos que caían de su interior eran las entrañas del animal.

13. También hubo una cautiva que se enamoró perdidamente del cacique que la había raptado y lo acompañó hasta el momento de su ejecución a mano de los militares argentinos (Sosa 2001, p. 204 s.).

14. Por ej. Maruja Huatatoca vive aún en la región (Dayuma, comunicación verbal, Toñampare, 2001).

15. El número entre corchetes indica el número de página del original.

16. Joaquina y una pareja indigena fueron atacados por diez huaorani, que asesinaron a la pareja y se llevaron a la niña (Blomberg 1996, p. 23 ss.).

17. Posiblemente la hija de Sam Souder, en cuya hacienda, situada en la desembocadura del río Arajuno en el Napo, vivía Joaquina.

18. Tidmarsh deduce que «barradero » (en vez de varadero, fondeadero) proviene de la palabra " barro ». Si bien etimológicamente incorrecta, el barrial y el lodo característicos en los varaderos del Oriente ecuatoriano hacen comprensible el error de Tidmarsh.

19. Tradicionalmente los casamientos son decididos por los ancianos y se realizan en ocasión de una fiesta (véase Rival 1996). El informe escrito por Cooper, citado en Blomberg (1996, p. 29), da como motivo de la huida que un niño, al que Joaquina cuidaba con otras mujeres, se ahogó en el río y ella temió las represalias.

20. No hallo en la bibliografía datos que sustenten esta afirmación. Quizás Joaquina interpreta como una costumbre huao lo que fue un suceso particular.

21. Llushan pishcu, llamado en otras áreas munami, es un ave pequeña de color azul claro, que hace su nido con algodón (Orr \& Wrisley 1981, p. 102).

22. Los signos de interrogación pertenecen al autor del documento.

23. Tachado : (escrito a máquina) " however Dr. Tschopp has one fitted with a handle which was found in an auca house », y finaliza, escrito a mano : " on a playa of the Misahuali ».

24. Se refiere posiblemente al libro de Tessmann, publicado en 1930.

25. Aquí no se trata de la expresión quichua para «niño », sino del nombre Wawae, mencionado por Wallis $(1996$, p. 37). Es posible identificar algunas personas del grupo a partir de las informaciones de Dayuma a los misioneros. Dayuma vivía aún allí cuando estuvo Joaquina : Wawae era su hermano mayor, Gikita su tío paterno, Karae su abuelo (Wallis 1996, pp. 37, 44).

26. Tía materna de Dayuma, según el árbol genealógico de Dayuma recogido por St. Beckerman (material de campo) y posiblemente también esposa de su padre (aquí aparece como " Mintara »). Más abajo se lee "Akao », seguramente Akawo, la madre de Dayuma. Gami o Ngami era su tía materna más joven, madre de Umi (Wallis 1996, pp. 87, 38, 37).

27. El hermano menor de Dayuma (Wallis 1996, p. 126). 


\section{BIBLIOGRAFÍA}

Altube, María Inés, 1999 - « Mujeres en "tierra adentro". Las cautivas en las sociedades indígenas de la región pampeana y nordpatagónica (siglos xviII y xIx) ", en : D. Villar, M. Herminia Di Liscia \& M. J. Caviglia (eds.), Historia y género. Seis estudios sobre la condición femenina, Universidad Nacional del Sur y Universidad Nacional de La Pampa, Buenos Aires.

Avendaño, Santiago, 2000. - Usos y costumbres de los indios de la Pampa, Buenos Aires.

Baumann, Peter \& Erwin Patzelt, 1982. - Menschen im Regenwald, Umschau, Frankfurt am Main.

Blomberg, Rolf, 1996. - Los aucas desnudos. Una reseña de los indios del Ecuador, Colección Tierra Incógnita, 20, Abya-Yala, Quito.

Cabodevilla, Miguel Angel, 1994. - Los huaorani en la historia de los pueblos del Oriente, Cicame, Coca.

CARVAJAl, Lino de, 1995. - «La cautiva o Rayhuemy ». Obra anotada por Maria E. Ginobili de Tumminello, Instituto Superior Juan xxIII y Fundación Ameghino, Bahía Blanca, Viedma (Argentina).

Cerón, M., Carlos y Consuelo Montalvo A., 1998. - Etnobotánica de los huaorani de Quehueiri-Ono, Napo (Ecuador), Abya-Yala, Quito.

Cipolletti, María Susana, 1998. - « La minuciosidad de la violencia y los mainas del Amazonas superior en un manuscrito del jesuita Juan Magnin (1742) ", en : S. DedenbachSalazar Sáenz et al., 50 Años de estudios americanistas en la universidad de Bonn. Nuevas contribuciones a la arqueología, etnohistoria, etnolinguistica y etnografia de las Américas, BAS, 30, pp. 449-469, Markt Schwaben.

Crivelli, Eduardo, 1997. — « El malón como guerra. El acoso a la frontera de Buenos Aires y la pacificación de las Pampas a finales del siglo XVIII ", en : M. S. Cipolletti (ed.), Resistencia y adaptación nativas en las tierras bajas latinoamericanas, Col. Abya-Yala, 36, Quito, pp. 175-204.

Dall'AlbA, Leonir, 1992. — « Relatos de colonos e indígenas sobre los Wao », en : G. Tassi (coord.), Náufragos del mar verde. La resistencia de los huaorani a una integración impuesta, Abya-Yala, Confeniae, Quito, pp. 95-124.

ELLıo, Elisabeth, 1981 [1956]. - Through Gates of Splendor, Wheaton, Ill.

—, 1989 [1961]. - The Savage my Kinsman, Ann Arbor, Mich.

Ferguson, R. Brian, 1999 [1990]. - "A Savage Encounter : Western Contact and the Yanomami War Complex », en : R. B. Ferguson \& N. Whitehead (eds.), War in the Tribal Zone. Expanding States and Indigenous Warfare, School of American Research Press, Santa Fe, New Mexico, pp. 199-228.

Hudelson, John E., 1984. — « The Lowland Quechua as "Tribe" ", en : Jeffrey Ehrenreich (ed.), Political Anthropology' of Ecuador, State University of New York at Albany, pp. 59-79.

Hux, Meinrado, 1999. - Memorias del ex cautivo Santiago Avendaño (1834-1874), Buenos Aires.

LÁzaro Avila, Carlos, 1994. - « Los cautivos en la frontera araucana », Revista española de antropología americana, 24, Editorial Complutense, Madrid, pp. 191-207. 
Muratorio, Blanca, 1987. - Rucuyaya Alonso y la historia social y económica del Alto Napo 1850-1950, Abya-Yala, Quito.

Oberem, Udo, 1980. - Los Quijos. Historia de la transculturación de un grupo indigena en el Oriente Ecuatoriano, Colección Pendoneros, 16, Instituto Otavaleño de Antropología, Otavalo.

Orywal, Erwin, 1996. - « Krieg als Konfliktaustragungssstrategie-Zur Plausibilität von Kriegsursachentheorien aus kognitionsethnologischer Sicht ", Zeitschrift für Ethnologie, 121, Berlín, pp. 1-48.

Orr, Carolyn y Betsy Wrislex, 1981. - Vocabulario quichua del Oriente, Vocabularios Indigenas, 11, Instituto Lingüístico de Verano, Quito.

Patzelt, Erwin, 1992. — Letzte Hoffinung Regenwald, Verlag Steiger, Innsbruck.

RivaL, Laura, 1993. - « The growth of familiy trees : understanding Huaorani perceptions of the forest ", Man, N.S., 28, pp. 635-652.

—, 1996a. - Hijos del Sol, padres del jaguar. Los huaorani de ayer y hoy, Biblioteca Abya-Yala, 35, Quito.

—, 1996b. — « Blowpipes and spears. The social significance of Huaorani technological choices », en : Philippe Descola \& Gisli Palsson (eds.), Nature and Society: Anthropological Perspectives, London-New York, pp. 145-164.

—, 1998. - " Androgynous parents and guest children : the Huaorani Couvade ", Journal of the Royal Anthropological Institution, N.S., 4, pp. 619-642, London.

—, 1999. — "The Huaorani », en : Richard B. Lee \& Richard Daly (eds.), The Cambridge Encyclopedia of Hunters and Gatherers, Cambridge University Press, Cambridge, Ma., pp. 101-104.

Robarcheк, Clayton \& Carole, 1998. - Waorani. The Contexts of Violence and War, Harcourt Brace College Publishers, Forth Worth.

Sosa, Norma, 2001. - Mujeres indigenas de la Pampa y Patagonia, Emecé, Buenos Aires.

Stoll, David, 1982. - Fishers of Men or Founders of Empire ? The Wycliffe Bible Translators in Latin America, Cultural Survival, Cambridge, Ma.

Tessmann, Günter, 1930. - Die Indianer Nordost-Perus, Hamburg 1930 [edición en español : Los indigenas del Perú Nordoriental, Ediciones Abya-Yala, Quito 1999]

Tidmarsh, Wilfred T., 1945. - Vocabulario tentativo del Idioma de los Aucas con unas Frases, Archivo Histórico del Banco Central, Quito, Manuscrito, Col. 24, 01411.

Tidmarsh, Wilfred T., \& Joaquina GreFa, 1945. - Unarranged Notes on the Aucas of the Nushino Basin, mecanografiado, 8 p. [transcripto en este articulo].

Valero, Helena, 1984. - Yo soy Napeyoma. Relato de una mujer raptada por los indigenas yanomami, Emilio Fuentes (ed.), Monografía 535, Fundación La Salle de Ciencias Naturales, Caracas.

Wallis, Ethel E., 1996 [1960]. - Dayuma. Life under Waorani Spears, YWAM Publishing, Seattle.

Whitten Jr., Norman E., 1976. - Sacha Runa. Ethnicity and Adaptation of Ecuadorian Jungle Quichua, University of Illinois Press, Urbana, Ill.

Yost, James A., 1981a. - «Los Waorani : Un pueblo de la selva », en : Acosta-Solís et al. (eds.), Ecuador: A la sombra de los volcanes, Quito, pp. 97-115.

—, 1981b. — « Twenty years of Contact : The Mechanisms of Change in Wao ("Auca") Culture ", en : Norman E. Whitten Jr. (ed.), Cultural Transformations and Ethnicity in Modern Ecuador, University of Illinois Press, Urbana, Ill., pp. 677-704. 
—, 1994. — "Waorani », en : Johannes Wilbert (ed.), Encyclopedia of World Cultures, 7, Prentice Hall International, London, pp. 351-354.

Yost, James A. \& Patricia Kelley, «Shotguns, Blowguns, and Spears: The Analysis of Technological Efliciency », en : Raymond B. Hames \& William T. Vickers (eds.), Adaptative Responses of Native Amazonians, pp. 189-224, Academic Press, New York.

Zerries, Otto, 1982. — « Die kulturgeschichtliche Stellung der Auka unter den Urwaldindianern Südamerikas », en : P. Baummann \& E. Patzelt, op. cit., pp. 158-162. 